\title{
A Simple and Fair Proposal to Improve the Performance of the IEEE 802.11e Enhanced Coordination Function
}

\author{
Marta Barría ${ }^{1}$, Pablo Sánchez ${ }^{2}$ and Luciano Ahumada ${ }^{3}$ \\ 1 Departamento de Computación, Universidad de Valparaíso, \\ Avenida Gran Bretaña 1091, Valparaíso, Chile. \\ 2 Departamento de Electrónica, Universidad Técnica Federico Santa María \\ Avenida España 1680, Valparaíso, Chile. \\ 3 Escuela de Ingeniería Informática, Universidad Diego Portales \\ Avenida Ejército 441, Santiago, Chile.
}

\begin{abstract}
A simple and fair proposal to improve the performance of the IEEE 802.11e standard is presented in this paper. Our proposal is accomplished by means of increasing the priority of those queues that have not been able to transmit during certain period of time, depending on the elapsed time waiting to transmit. Results show that this proposal improves the performance of wireless networks using IEEE 802.1 le EDCF since low priority queues reduce their waiting time to access the channel and high priority queues are not degraded.
\end{abstract}

\section{Introduction}

Wireless Local Area Networks (WLAN) are gaining popularity at public hot spots, home and work. To provide an efficient and robust network in a wireless environment for a collection of mobile stations, the IEEE 802.11 working group has chosen the Carrier Sense Multiple Access with Collision Avoidance (CSMA/CA) protocol as the standard protocol [1]. This standard contains two access methods: the distributed coordination function (DCF) and point coordination function (PCF), with the former being specified as the fundamental method. Notice that they can only support best effort traffic [2].

With the widespread use of wireless services and the emerging requirements of real-time voice, audio and multimedia applications, Quality of Service (QoS) support becomes a key requirement. To address this challenge, the IEEE 802.11 Task Group E published the IEEE 802.11e draft [3], presenting the enhanced distributed coordination function (EDCF) and the hybrid coordination function (HCF) providing

Please use the following format when citing this chapter:

Barría, M., Sánchez, P., Ahumada, L., 2006, in IFIP International Federation for Information Processing, Volume 211, ed. Pujolle, G., Mobile and Wireless Communication Networks, (Boston: Springer), pp. $123-131$. 
differentiated services in terms of priority schemes to access the medium for different stations or traffic nature. The EDCF assures the best service for high priority traffic and minimum service for those of low priority. Although this mechanism improves the performance of a wireless real time traffic service, it may not be a fair scheme for those queues of medium or low priority since the EDCF parameters are not adapted to the network status and low or medium priority traffic may not be treated fairly since they could suffer starvation or extremely high latency.

In this work we present a novel method to improve the performance of the IEEE 802.11e EDCF, being fair with all traffic categories and simpler than other proposals [2]. Different changes to the IEEE 802.11e have been proposed. However, most of them only care about high priority queues, sacrificing ACs of low or medium priority. Thus, if the performance of those models is evaluated in terms of a fair scheme, they seem to be not appropriated enough. To achieve fairness, fair scheduling mechanisms have been proposed for WLANs. Among them, the Distributed Fair Scheduling (DFS) tries to adjust backoff intervals [6], Distributed Weighted Fair Queuing (DWFQ) [7] adjusts contention windows, and Distributed Deficit Round Robin (DDRR) [8] suggests the use of different inter-frame space (IFS) intervals through a mapping scheme to avoid possible collisions. Distributed Elastic Round Robin (DERR) [9] determines an allowance value according to users' requirements. Both DFS and DWFQ present poor performance in terms of the throughput and delay due to collisions although better fairness can be achieved. DDRR presents better performance in throughput and delay than DFS and DWFQ; however DERR not only improves performance in throughput and delay, but also exhibits better fairness if it is compared to DDRR [9].

We aim to establish a simple methodology by the estimation of simple parameters in each station, without the knowledge of the entire network or the state of the shared wireless medium. Our technique is based on considering that all stations are statistically similar, that is, traffic arrival to each priority queue is described by the same Poisson process. Therefore, looking for fairness at each station will create a fair network.

The rest of this article is presented as follows: in section 2 we describe the IEEE 802.11 DCF and 802.11e EDCF, section 3 contains the proposal, section 4 the performance evaluation and simulation results and finally in section 5 we present our conclusions.

\section{Brief Description of IEEE 802.11 DCF and 802.11e EDCF}

\subsection{The IEEE 802.11 Distributed Coordination Function}

The IEEE 802.11 standard [1] defines both the physical (PHY) and medium access control (MAC) layer protocols for WLANs. The IEEE 802.11 standard calls for three different PHY specifications: frequency hopping (FH) spread spectrum, direct sequence (DS) spread spectrum, and infrared (IR).

The basic data rate for the DS system is $1 \mathrm{Mbits} / \mathrm{s}$ encoded with differential binary phase shift keying (DBPSK). Similarly, a $2 \mathrm{Mbits} / \mathrm{s}$ rate is provided using differential quadrature phase shift keying (DQPSK) at the same chip rate. Higher 
rates of 5.5 and $11 \mathrm{Mbits} / \mathrm{s}$ are also available using techniques combining quadrature phase shift keying and complementary code keying (CCK); all of these systems use $22 \mathrm{MHz}$ channels. The IEEE 802.11 MAC layer specifications, common to all PHYs and data rates, coordinate the communication between stations and control the behavior of users who want to access the network.

According to IEEE 802.11 standard stations access the channel using a basic access method, or an optional four-way handshaking access method with an additional Request-To-Send/Clear-To-Send (RTS/CTS) message exchange. Under the basic access method, a station, when ready for a new data frame transmission, first senses the channel status. If the channel is found to be busy, the station defers its transmission and continues to sense the channel until it is idle. After the channel is idle for a specified period of time called the distributed interframe space (DIFS) period, the station defers its transmission and continues to sense the channel until it is idle. If the medium is sensed idle for a period greater than a DCF Interframe Space (DIFS), the station goes into a backoff procedure before it sends its frame. Upon the successful reception of a frame, the destination station returns an ACK frame after a Short Interframe Space (SIFS). If an ACK is not received within an ACK timeout interval, the station assumes that either the data frame or the $\mathrm{ACK}$ was lost and needs to retransmit its data frame by repeating the basic access procedure.

The backoff procedure shall be invoked by the station when the medium is sensed busy as indicated by either the physical or CSMA/CA algorithm. To begin the backoff procedure, the station shall set its Backoff Timer to a random backoff time. All backoff slots occur following a DIFS period during which the medium is determined to be idle for the duration of the DIFS period. Note that the time immediately after the DIFS period is slotted. The timeslot duration is at least the time required for a station to detect an idle channel plus the time required for switching from listening to transmitting mode. The backoff window is based on a random value uniformly distributed in the interval [CWmin; CWmax], where CWmin and CWmax represent the Contention Window parameters. The backoff timer is decreased by one for each idle slot, stopped if the channel is sensed busy, and then reactivated if the channel is idle again and remains idle for more than a DIFS time duration. When the backoff timer reaches zero, the data frame is transmitted.

A virtual carrier sense mechanism is also provided at the MAC layer. It uses the request-to-send (RTS) and clear-to-send (CTS) message exchange to make predictions of future traffic on the medium and updates the network allocation vector (NAV) available in stations. Communication is established when one of the wireless nodes sends a short RTS frame. The receiving station issues a CTS frame that echoes the sender's address. If the CTS frame is not received, it is assumed that a collision occurred and the RTS process starts over. The RTS/CTS scheme is designed to avoid the so-called Hidden Terminal problem [1], which occurs when mobile stations are unable to hear each other.

Typically, time-bounded applications such as Voice over IP (VoIP), or videoconferencing require specified bandwidth, low delay and jitter, but can tolerate some losses. The point is that in DCF, all the stations compete for the channel with the same priorities. There is no differentiation mechanism to guarantee bandwidth, packet delay and jitter for high-priority multimedia flows [2]. If this is added to 
channel fading [4], it results simple to understand that the DCF may not be the best scheme to support QoS oriented services.

\subsection{The IEEE 802.11e Enhanced Distributed Coordination Function}

The EDCF enhances the DCF. It provides distributed and differentiated channel access for frames with 8 different priorities. It is a part of a single coordination function, called the Hybrid Coordination Function (HCF), of the 802.11e MAC specifications. All the details of the HCF [3] are beyond the scope of this paper as we focus on the EDCF.

Data frames from the higher layers, with a specific priority value, arrive at the MAC layer. Then, each QoS data frame carries its priority value in the MAC frame header. An 802.11e station shall implement four access categories (ACs), mapping the arrived frames to a specific AC queue according to a predefined mapping table, as shown in Table 1 . This is usually extracted from IEEE $802.1 \mathrm{~d}$ bridge specification [5].

Table 1. AC Mapping Table

\begin{tabular}{ccc}
\hline Priority & AC & Traffic Type \\
\hline 0 & 0 & Best effort \\
1 & 0 & Best effort \\
2 & 0 & Best effort \\
3 & 1 & Video Probe \\
4 & 2 & Video \\
5 & 2 & Video \\
6 & 3 & Voice \\
7 & 3 & Voice \\
\hline
\end{tabular}

The AC queues differ from each other on the specific value of the initial length of the contention window $\mathrm{CW}[\mathrm{AC}]_{0}$. This means that high priority traffic will be described in terms of a smaller backoff interval than the corresponding value for those of low priority.

Each AC in a station will sense the channel and it will start an independent backoff procedure after an arbitrary inter-frame space (AIFS) during which the medium is determined to be idle. The AIFS value may be different for each AC, i.e. AIFS[AC] may differ from each other. The minimum value of AIFS[AC] is DIFS. After an AIFS period, each backoff timer will take a random value in the interval $\left[1 ; \mathrm{CW}[\mathrm{AC}]_{0}+1\right]$.

The backoff timer of each AC is decreased by one for each idle slot, stopped if the channel is sensed busy, and then reactivated if the channel is idle again and remains idle for more than an AIFS time duration. When the backoff timer reaches zero, the data frame is transmitted.

When there is more than one AC finishing the backoff at the same time, the collision is handled in a virtual manner by a virtual collision handler. That is, the highest priority frame among the colliding frames receives a transmission opportunity (TXOP) and the others perform a backoff with increased $\mathrm{CW}$ values, 
whose size will be given by their previous value $\mathrm{CW}[\mathrm{AC}]$ and a persistence factor (PF), according to:

$$
\mathrm{CW}[\mathrm{AC}]_{\mathrm{i}}=\left(\mathrm{CW}[\mathrm{AC}]_{\mathrm{i}-1}+1\right) \cdot \mathrm{PF}[\mathrm{AC}]-1 \quad \mathrm{i}>0
$$

Therefore, the backoff timer will take a random value in the interval $\left[1 ; \mathrm{CW}[\mathrm{AC}]_{\mathrm{i}}+1\right]=\left[1 ;\left(\mathrm{CW}[\mathrm{AC}]_{\mathrm{i}-1}+1\right) \cdot \mathrm{PF}[\mathrm{AC}]\right]$. Notice that this procedure does not avoid colliding packets from different stations to access the shared physical medium.

\section{The TBPS scheme}

The EDCF is a non adaptive protocol, since the priority of each AC is static. Therefore, if the offered load is high, certain traffic classes may experience starvation because of an increasing number of virtual collisions, being an unfair scheme for those ACs of low or medium priority. If we also take into account collided packets from different stations, or a non-ideal wireless channel, the problem is even worst.

In this work we propose the use of a dynamic priority scheme for the $\mathrm{AC}$ queues, named Time Based Priority Scheme (TBPS). Our proposal is to update the ACs priorities depending on the waiting time to transmit a frame of each queue. Hence, if any $\mathrm{AC}$ queue has not been able to transmit a frame because of virtual collisions, i.e. the scheduler mechanism of the virtual collision handler, its priority is increased allowing it to transmit a frame in a number of retrievals not bigger than the number of working queues in that station. This change of priority implies that if a virtual collision occurs, the "virtually collided" AC with longer waiting time interval must use the AIFS and CW values of the queue with immediately higher priority.

This scheme establishes a hard transmission limit. After a successful transmission attempt, each AC must load their original values of CW and AIFS. Then, the QoS of each $\mathrm{AC}$ will be a function of its own initial contention window $\mathrm{CW}[\mathrm{AC}]_{0}$ and $\mathrm{AIFS}[\mathrm{AC}]$ periods guaranteeing that a single $\mathrm{AC}$ will always be able to use the channel in a number of retrievals not bigger than the number of working queues in that station. Therefore, faimess is assured by means of a simple method that only requires the estimation of local parameters, being less complex than those that need a complete knowledge of the network.

\section{Performance Evaluation and Simulation Results}

We based our simulations design on the event-oriented software presented in [10]. Our simulations have a confidence interval of $95 \%$. Simulated stations used three different $\mathrm{AC}$ queues: $\mathrm{AC} 3$ with high priority (HP), $\mathrm{AC} 2$ with medium priority (MP) and $\mathrm{AC} 1$ with low priority (LP). If a virtual collision occurs, the classic EDCF would let $\mathrm{AC} 3$ be the first station to transmit, and $\mathrm{AC} 1$ the last. Our dynamic proposal suggests that the order will depend on the elapsed time by each $\mathrm{AC}$ waiting to transmit a frame. This implies that if a virtual collision happens, the queue that has 
been waiting to transmit for a longer period of time must replace its $\mathrm{CW}[\mathrm{AC}]$ and AIFS[AC] values taking those of the following queue with higher priority. For example, if $\mathrm{AC} 3$ is the queue being on hold for a longer period of time, it must replace its $\mathrm{CW}$ and AIFS values taking those of $\mathrm{AC} 2$. After a successful transmission attempt, each $\mathrm{AC}$ must load their original values of $\mathrm{CW}$ and AIFS.

Once the virtual collision handler of each station selects an $\mathrm{AC}$ queue to transmit, it may sense an idle or busy channel. If the channel is idle, data frames may collide with those of another station trying to transmit or start a successful transmission. During our simulations, we analyze the performance of this proposal in terms of the busy channel probability, defined as the probability that the shared wireless medium is not idle. We use a unique packet length for all ACs and stations. We normalize packet and CW lengths to the slot lengths defined by the PHY layer. The defaults settings are described in Table 2.

Table 2. Default settings to run the simulations

\begin{tabular}{llll}
\hline Parameter & AC3 & AC2 & AC1 \\
\hline Priority & HP & MP & LP \\
AIFS & 4 slots & 5 slots & 6 slots \\
CWmin & 8 slots & 16 slots & 32 slots \\
PF & 2 & 2 & 2 \\
Packet length & 38 slots & 38 slots & 38 slots \\
\hline
\end{tabular}

We evaluate the network performance in terms of the latency and mean occupation of the network. Latency is defined as the time interval for a packet to come into their queue until it accesses the medium. The mean occupation of each AC is the ratio between the successfully used slots (without collisions) and the entire number of time slots.

\subsection{Simulation Results}

Figure 1 shows that if the busy channel probability is increased, the mean occupation of each AC is significantly decreased since the backoff is continuously interrupted and the probability of finding an idle channel for a period longer than AIFS is also diminished. Notice that AC3 always presents higher occupation, since it has higher priority. It can also be seen that the dynamic priority method improves the performance of $\mathrm{AC2}$ and $\mathrm{ACl}$ as compared to the static mechanism, without a significant change on the performance of AC3.

Figure 2 shows the latency results. It can be seen that the latency is reduced for low and medium priority queues $\mathrm{AC} 2$ and $\mathrm{AC} 1$, and $\mathrm{AC} 3$ does not present significant changes. This improvement makes the dynamic approach a better scheme if the offered load is high or if the network is saturated since QoS requirements may be achieved for all queues. 


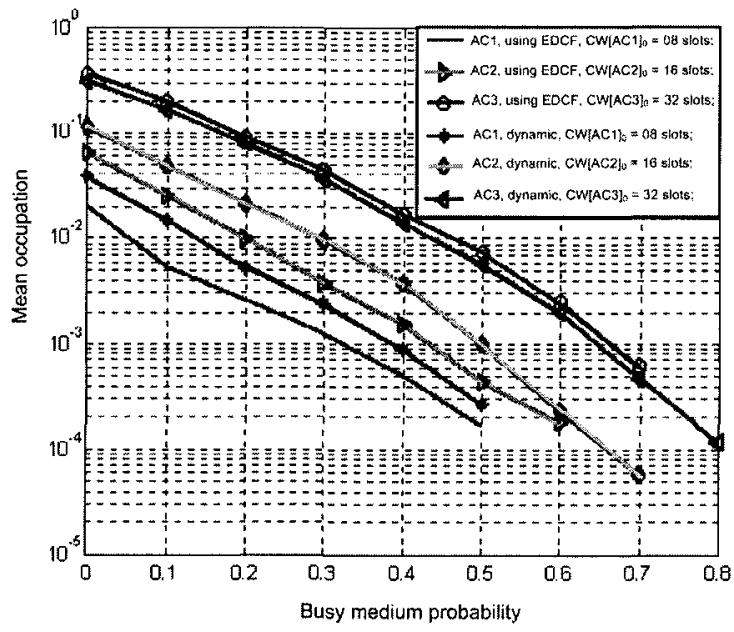

Fig. 1. Mean occupation in terms of the busy medium probability

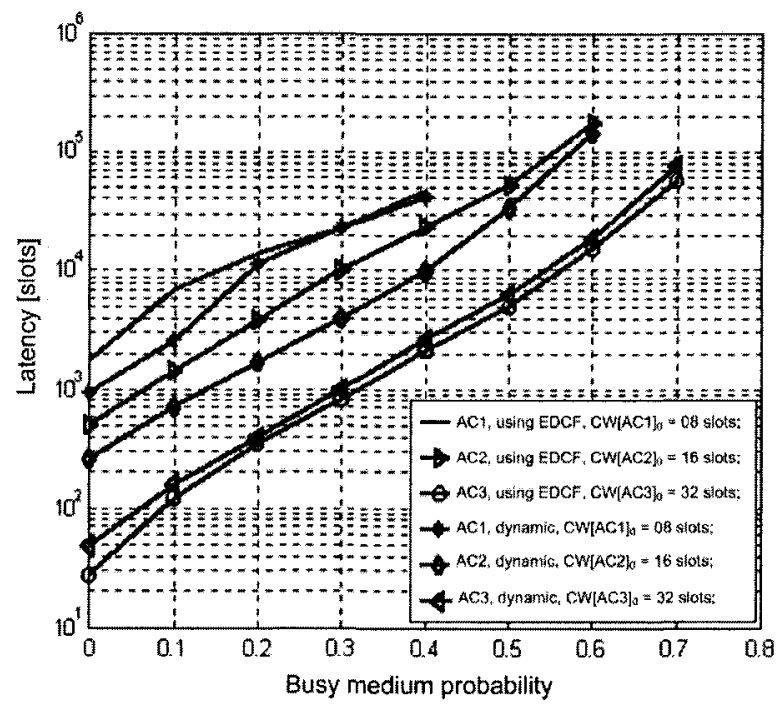

Fig. 2. Latency in terms of the busy medium probability 


\section{Conclusions}

The main contribution of this article is the novel mechanism to handle virtual collisions as an upgrade of the IEEE 802.11e EDCF. This method is simpler than those already published since it only requires the estimation of local parameters, i.e. parameters from the same station. This dynamic methodology improves the performance of low priority queues, maintaining the performance of high priority ACs. Simulations results show that the use of this proposal makes significant improvements on latency and occupancy.

\section{Acknowledgments}

This project was partially supported by project DIPUV-48/2005 and project Semilla-UDP.

\section{References}

1. IEEE 802.11 WG, Reference number ISO/IEC 8802 -11:1999(E) IEEE Std. 802.11, 1999 edition. International STANDARD for Information TechnologyTelecommunications and information exchange between Systems Local and Metropolitan area networks- Specifics requirements- Part 11: Wireless LAN Medium Access Control (MAC) and Physical Layer (PHY) Specifications, (1999).

2. Huang Zhu, Ming Li, Imrich Chlamtac, and B. Prabhakaran, "A Survey of Quality of Service in IEEE 802.11 networks", IEEE Wireless Communications Magazine. 11(4) 4-14 (2004).

3. IEEE 802.11 WG, Draft Supplement to standard for Telecommunications and Information Exchange between Systems LAN/MAN Specific RequirementsPart 11: Wireless Medium Access control and Physical Layer (PHY) Specifications: Medium Access control (MAC) Enhancements for Quality of Services (QOS), IEEE 802.11e/ D2.0, (2001).

4. L. Ahumada, R. Feick, R. A. Valenzuela and C. Morales: Measurement and Characterization of the Temporal Behavior of Fixed Wireless Links. IEEE Transactions on Vehicular Technology. 54(6) 1913-1922 (2005).

5. IEEE 802.1d-1998, Part 3: Media Access Control (MAC) bridges, ANSI/IEEE Std. 802.1D, (1998)

6. N. H. Vaidya, P. Bahl, and S. Gupta, "Distributed fair scheduling in a wireless LAN," in Proc. ACM MobiCom '00. 167-178 (2000).

7. Banchs and X. Perez, "Distributed weighted fair queuing in 802.11 wireless LAN," in Proc. IEEE ICC '02. 3121-3127 (2002).

8. W. Pattara-Atikom, S. Banerjee, and P. Krishnamurthy, "Starvation prevention and quality of service in wireless LANs," in Proc. IEEE Wireless Personal Multimedia Communications '02. 1078-1082 (2002). 
9. Huei-Wen Ferng, Chung-Fan Lee, Jeng-Ji Huang, and Ge-Ming Chiu, "Designing a Fair Scheduling Mechanism for IEEE 802.11 Wireless LANs" IEEE Communications Letters, 9(4) 301-3003 (2005).

10. S. Mangold, and S. Choi. "IEEE 802.11e Wireless LAN for Quality of Service". European Wireless (EW'02). 32-39 (2002). 